\title{
Exhaustive generation for permutations avoiding a (colored) regular sets of patterns
}

\author{
Phan Thuan Do * $\quad$ Thi Thu Huong Tran ${ }^{\dagger} \quad$ Vincent Vajnovszki ${ }^{\ddagger}$ \\ September 18, 2018
}

\begin{abstract}
Despite the fact that the field of pattern avoiding permutations has been skyrocketing over the last two decades, there are very few exhaustive generating algorithms for such classes of permutations. In this paper we introduce the notions of regular and colored regular set of forbidden patterns, which are particular cases of right-justified sets of forbidden patterns. We show the (colored) regularity of several sets of forbidden patterns (some of them involving variable length patterns) and we derive a general framework for the efficient generation of permutations avoiding them. The obtained generating algorithms are based on succession functions, a notion which is a byproduct of the ECO method introduced in the context of enumeration and random generation of combinatorial objects by Barcucci et al. in 1999, and developed later by Bacchelli et al. in 2004, for instance. For some classes of permutations falling under our general framework, the corresponding counting sequences are classical in combinatorics, such as Pell, Fibonacci, Catalan, Schröder and binomial transform of Padovan sequence.
\end{abstract}

Keywords : pattern avoiding permutation, right-justified forbidden pattern, exhaustive generating algorithm, succession function, ECO method.

\section{Introduction}

Given a class of combinatorial objects, it is a common problem to list exhaustively (with no repetitions nor omissions) all the objects with a given size in the class. Exhaustive generation can be used to test hypotheses about a class of objects, to support a conjecture or find counterexamples, to analyze or prove programs, etc, and often an exhaustive generating algorithm exhibits new properties of the class under consideration. Two books, that of F. Ruskey [12] and more recently that of D. Knuth [11] are entirely devoted to the exhaustive generation of combinatorial objects.

When generating combinatorial objects, the time complexity of a generating algorithm is crucial since the cardinality of a class is, in general, an exponential function of the size

\footnotetext{
${ }^{*}$ Department of Computer Science, Hanoi University of Science and Technology, 01 Dai Co Viet, Hanoi, Vietnam, Email: thuandp@soict.hust.edu.vn

${ }^{\dagger}$ Vietnamese-German University, Le Lai street, Hoa Phu ward, Thu Dau Mot city, Binh Duong, Vietnam, Email: huong.ttt@vgu.edu.vn

${ }^{\ddagger}$ LE2I, Université de Bourgogne Franche-Comté, B.P. 47 870, 21078 Dijon-Cedex, France, Email: vvajnov@u-bourgogne.fr
} 
of the generated objects. If a generating algorithm produces combinatorial objects so that only a constant amount of computation is done between successive objects, in an amortized sense, then one says that it runs in constant amortized time (or CAT) [12].

The field of pattern avoiding permutations has been showing an increasing interest in the last two decades. However little has been done so far on the exhaustive generation of such classes of permutations. A very powerful way to define, enumerate and construct recursively such permutation classes is the ECO method [1, 3. This is a general recursive description of combinatorial classes which explains how an object of a given size can be reached uniquely from an object of smaller size. More specifically, the ECO method specifies through a succession function how many objects of larger size can be obtained from an object of a given size.

In this paper, which is an enhanced version of the conference version [7, we give a general framework for the efficient (that is, CAT) exhaustive generation of some classes of pattern avoiding permutations. The classes under consideration are defined by means of regular and colored regular set of forbidden patterns, both defined in this paper. They cover a wide range of pattern avoiding permutations and many of them are enumerated by classical integer sequences. A main ingredient for our generating algorithms is the succession function corresponding to the set of forbidden patterns.

After the presentation of some basic definitions at the end of this section, the remainder of the paper is structured as follows. In Section 2 we recall the notion of right-justified set of forbidden patterns, originally introduced in [6, 8, and we give a characterization of such sets of patterns. Section 3 is devoted to regular sets of forbidden patterns, a subclass of right-justified ones where the succession functions are computationally efficient. We show that a particular set of forbidden patterns involving two variable length patterns is regular, and some instances of it yield known counting sequences for the corresponding pattern avoiding permutations. This notion is further refined in Section 4 to colored regular sets of forbidden patterns, and we show the colored-regularness of some sets of forbidden patterns (one of them involving a variable length pattern) and, as previously, some known enumerating sequences are obtained. Notice that the idea of color labeling in the ECO generating context has previously been mentioned in [2, 4]. In the last section we present a general framework for the efficient exhaustive generation for permutations avoiding a regular or a colored regular set of forbidden patterns. Finally, in Appendix, we give a list of regular and colored regular sets of forbidden patterns together with their succession functions. Each of these classes can be exhaustively generated in constant amortized time by our algorithms.

\section{Pattern avoiding permutations}

We denote by $S_{n}$ the set of permutations on $\{1,2, \ldots, n\}, n \geq 0$, and the empty permutation $\epsilon$ is the unique permutation in $S_{0}$. We use the one-line notation: for $\pi \in S_{n}$ we write $\pi=\pi(1) \pi(2) \ldots \pi(n)$, where $\pi(i)$ is the image of $i$ by $\pi$, and $n$ is said the length of $\pi$. A permutation $\sigma$ is contained in another permutation $\pi$ if $\pi$ has a (not necessarily contiguous) subsequence whose terms are order isomorphic to (i.e., have same relative ordering as) $\sigma$. In this context $\sigma$ is called a pattern, and if $\sigma$ is not contained in $\pi$ we say that $\pi$ avoids $\sigma$. For example the permutation $461532 \in S_{6}$ contains the pattern 312 because the sequence 413 (among others) is ordered in the same way as 312 , whereas $24531 \in S_{5}$ 
avoids 312 .

For a set of patterns $P$ we say that a permutation avoids $P$ (or, it is $P$-avoiding), if it avoids each pattern in $P$, and in this context $P$ is called set of forbidden patterns. We denote by $S_{n}(P)$ the set of length $n$ permutations avoiding $P$ :

$$
S_{n}(P)=\cap_{\sigma \in P}\left\{\pi \in S_{n}: \pi \text { avoids } \sigma\right\},
$$

and $S(P)=\cup_{n \geq 0} S_{n}(P)$. The set $S(P)$ is a downset in the permutation pattern involvement order, that is, $\pi$ belongs to $S(P)$ whenever $\pi$ occurs as a pattern in a permutation in $S(P)$. See S. Kitaev's seminal book [10] for an extensive presentation of pattern avoidance in permutations.

\section{Right-justified forbidden patterns}

Here we introduce the right-justified forbidden patterns, a particular class of forbidden patterns defined in [6, 8], and we give a characterization of them. In the next two sections we refine this notion to (colored) regular patterns.

Let $P$ be a set of forbidden patterns. Each permutation in $S_{n}(P), n \geq 1$, can be obtained from a unique one in $S_{n-1}(P)$ by inserting the entry $n$ into the appropriate position. Informally, $P$ is said to be right-justified if, for any $n \geq 1$, it satisfies the following property: if the insertion of $n$ into the position $i$ of $\alpha \in S_{n-1}(P)$ yields a permutation in $S_{n}(P)$, then so does the insertion of $n$ into any position to the right of $i$ in $\alpha$.

Let $\alpha$ be a length $n$ permutation. We denote by $\alpha^{\rightarrow}$ (resp. $\alpha^{\leftarrow}$ ) the permutation obtained from $\alpha$ by moving its largest entry $n$ to the right (resp. left) one position; and $\alpha^{\rightarrow}\left(\right.$ resp. $\left.\alpha^{\leftarrow}\right)$ is defined only if $\alpha(n) \neq n$ (resp. $\alpha(1) \neq n$ ). For instance, $3142^{\rightarrow}=3124$ and $3142^{\leftarrow}=3412$.

Definition 1. The set of forbidden patterns $P$ is said to be right-justified if $\alpha \in S(P)$ implies $\alpha \rightarrow \in(P)$.

In other words, $P$ is right-justified if for any length $n$ permutation avoiding $P$ by moving $n$ to the right we still obtain a permutation avoiding $P$.

\section{Example 1.}

- $P=\{132\}$ is not right-justified since, for instance, $3412 \in S_{4}(132)$ but $3412 \rightarrow=$ $3142 \notin S_{4}(132)$. It is easily seen that $P=\{312\}$ is right-justified, and in general, a singleton set of forbidden patterns $P=\{\tau\}$ with $\tau \in S_{k}$ is right-justified if and only if $\tau(1)=k$.

- $P=\{312,123\}$ is not right-justified since, for instance, $132 \in S_{3}(P)$ but $132^{\rightarrow}=$ $123 \notin S_{3}(P)$. By contrast, $P=\{312,132\}$ is right-justified and Theorem 11 below gives a characterization of right-justified forbidden patterns.

The right-justifiedness of a set of forbidden pattern is a prerequisite for its regularity, a notion introduced in [8] and presented in the next section. Many permutation patterns considered in the literature are regular, although their right-justifiedness was considered only implicitly or even omitted. The next theorem gives a characterization of right-justified patterns. 
Theorem 1. The set $P$ of (possibly different lengths) forbidden patterns is right-justified if and only if for any $\tau \in P$, if $\tau^{\leftarrow}$ exists, then it contains a pattern in $P$.

Proof. Assume that $P$ is a set of right-justified forbidden patterns. Let $\tau \in P$ and suppose that $\tau^{\leftarrow}$ exists and it avoids $P$. Since $P$ is right-justified, we have $\left(\tau^{\leftarrow}\right) \rightarrow=\tau$ avoids $P$, which is a contradiction.

Conversely, let $P$ be a set of forbidden patterns such that $\tau^{\leftarrow}$ contains a pattern in $P$ for any $\tau \in P$, whenever $\tau^{\leftarrow}$ exists. We need to prove that $\alpha \rightarrow \in S_{n}(P)$ for any $\alpha \in S_{n}(P)$ whenever $\alpha \rightarrow$ exists. Let $\alpha \in S_{n}(P)$ and let $i<n$ be such that $\alpha(i)=n$. Thus, by definition

$$
\alpha^{\rightarrow}=\alpha(1) \ldots \alpha(i-1) \alpha(i+1) n \ldots \alpha(n),
$$

and let suppose that $u=u_{1} u_{2} \ldots u_{k}$ is an occurrence of the length $k$ pattern $\tau \in P$ in the permutation $\alpha \rightarrow$. We distinguish two cases.

If $u$ does not contain simultaneously entries $\alpha(i+1)$ and $n$, then $u$ is a subsequence of $\alpha$ too, so $\alpha$ contains $\tau$, which is a contradiction.

Otherwise, let $u^{\prime}$ be the sequence obtained from $u$ by transposing $\alpha(i+1)$ and $n$, that is, $u^{\prime}$ has the form $u_{1} u_{2} \ldots n \alpha(i+1) \ldots u_{k}$. Clearly, $u^{\prime}$ is an occurrence of $\tau^{\leftarrow}$ in $\alpha$, so $\alpha$ contains $\tau^{\leftarrow}$, which in turn contains a pattern in $P$, and thus $\alpha \notin S_{n}(P)$. This yields again to a contradiction.

In particular, if the patterns of $P$ have the same length, then we have the next simpler characterization.

Corollary 1. Let $P$ be a set of forbidden patterns of same length. Then $P$ is right-justified if and only if for each $\tau \in P$ we have $\tau^{\leftarrow} \in P$, whenever $\tau^{\leftarrow}$ exists.

Below are several examples of sets of forbidden patterns whose right-justifiedness follows directly from Theorem 1. Some of these patterns will be considered in more details in the next two sections.

\section{Example 2.}

1. $P=\{321,231\}$, and $\left|S_{n}(P)\right|=2^{n-1}$ for $n \geq 1$ (sequence A000079 in OEIS [13]).

2. $P=\{321,3412\}$ and $P=\{312,2431\}$, and for both $\left|S_{n}(P)\right|$ gives the bisection of Fibonacci sequence (A001519 in OEIS [13]).

3. $P=\{2134,2143,2413,4213\}$, and $\left|S_{n}(P)\right|$ gives the central binomial coefficients $\left(\begin{array}{c}2 n-2 \\ n-1\end{array}\right)$ (sequence A000984 in OEIS [13]).

4. $P=\{312,321,23 \ldots(p+1) 1\}, p \geq 2$, and $\left|S_{n}(P)\right|$ gives the sequence of $p$-generalized Fibonacci numbers. When $p=2, P$ becomes $\{312,321,231\}$ and $\left|S_{n}(P)\right|$ gives the sequence of Fibonacci numbers (A000045] in OEIS [13]), see for instance [2, 4].

5. $P=\{312,2431,(p+1) p \ldots 21\}, p \geq 2$. When $p=3, P$ becomes $\{312,2431,4321\}$ and $\left|S_{n}(P)\right|$ is the binomial transform of Padovan sequence (A034943 in OEIS [13]), see [4].

6. $P=\{321, p(p+1) 12 \ldots(p-1),(m+1) 12 \ldots m\}, p, m \geq 2$. In particular, 
- when $p=2, P=\{231,321,(m+1) 12 \ldots m\}$, and $\left|S_{n}(P)\right|$ gives again the generalized Fibonacci sequence. In particular, if $m=2$, then $P=\{231,312,321\}$ and as above $\left|S_{n}(P)\right|$ gives the sequence of Fibonacci numbers [2];

- when $p=m=3, P=\{321,3412,4123\}$, and $\left|S_{n}(P)\right|$ gives the sequence of Pell numbers (A215928 in OEIS [13]), see for instance [2, 9].

\section{Regular patterns}

Let $P$ be a set of right-justified forbidden patterns and $\alpha \in S_{n}(P)$. As we have seen in the previous section, if the insertion of $(n+1)$ into the $i$ th position of $\alpha$ yields a permutation in $S_{n+1}(P)$, then so does the insertion of $(n+1)$ into any position to the right of $i$. In order to formalize this phenomenon, we define a site of a permutation as a position between two of its entries, and before the first and after the last entries. Sites are numbered from right to left, and so the rightmost site, that which follows the last entry of the permutation, is numbered by one. And by convention, the length zero permutation $\epsilon$ has one site (numbered by one).

For $\alpha \in S_{n}$, we denote by $\alpha^{\downarrow i}$ the permutation obtained from $\alpha$ by inserting $(n+1)$ into its $i$ th site. For a set of forbidden patterns $P$ and $\alpha \in S_{n}(P)$, a site $i$ of $\alpha$ is called active (with respect to $P$ ) if $\alpha^{\downarrow i} \in S_{n+1}(P)$. For a set $P$ of right-justified forbidden patterns active sites of a permutation $\alpha \in S(P)$ form an interval of integers beginning by 1 , and if $i$ is an active site of $\alpha \in S(P)$ we denote by $\chi_{P}(i, \alpha)$ the number of active sites of $\alpha^{\downarrow}$. It follows that if $\beta=\alpha^{\downarrow i} \in S_{n+1}(P)$ for some $\alpha \in S_{n}(P)$ and an active site $i$ of $\alpha$, then $\beta^{\downarrow j} \in S_{n+2}(P)$ if and only if $j$ belongs to the interval $\left[1, \chi_{P}(i, \alpha)\right]$; and any permutation in $S_{n+2}(P)$ can be obtained uniquely in this way from appropriate $\alpha, i$ and $j$.

It can happen that $\chi_{P}(i, \alpha)$ does not depend on $\alpha$ but only on the number of active sites of $\alpha$, and we have the next definition.

Definition 2 (Regular pattern/succession function). A set $P$ of right-justified forbidden patterns is called regular if for any $\alpha \in S_{n}(P), n \geq 0$,

- $\alpha$ has its first site active, and

- if $\alpha$ has $k$ active sites, then for any $i, 1 \leq i \leq k$, the number $\chi_{P}(i, \alpha)$ of active sites of $\alpha^{\downarrow i}$ does not depend on $\alpha$ but solely on $i$ and on $k$. In this case, $\chi_{P}(i, \alpha)$ is denoted by $\chi_{P}(i, k)$, and

$$
\chi_{P}:\left\{(i, k) \mid k \in \mathbb{N}^{+}, 1 \leq i \leq k\right\} \rightarrow \mathbb{N}^{+}
$$

is called a succession function.

Even we will not use explicitly later, it is worth to mention that for regular sets of forbidden patterns $P$ characterized by the succession function $\chi_{P}$, the set of productions

$$
\left\{(k) \rightsquigarrow\left(\chi_{P}(1, k)\right)\left(\chi_{P}(2, k)\right) \ldots\left(\chi_{P}(k, k)\right)\right\}_{k \geq 1}
$$

is called succession rule corresponding to $P$. These productions are the core of ECOmethod introduced in [1] and were widely used afterwards in more general contexts, as the enumeration or (random and exhausive) generation of combinatorial objects.

The following theorem gives the succession function for the set of right-justified forbidden patterns in Example 26, and thus shows that it is a set of regular such patterns. 
Theorem 2. The succession function corresponding to $P=\{321, p(p+1) 12 \ldots(p-1),(m+$ 1) $12 \ldots m\}, p, m \geq 2$, is defined by:

$$
\chi_{P}(i, k)= \begin{cases}k+1, & \text { if } i=1 \text { and } k<m \\ m, & \text { if } i=1 \text { and } k=m \\ i, & \text { if } 1<i<p \\ p-1, & \text { otherwise. }\end{cases}
$$

Proof. Let $\alpha=\alpha(1) \alpha(2) \ldots \alpha(n)$ be a permutation in $S_{n}(P)$ with $k$ active sites. We consider the number of active sites of $\alpha^{\downarrow i}$ for each $i, 1 \leq i \leq k$, and since $P$ is rightjustified, this number is precisely the maximum (i.e., the number of the leftmost) site $j$ of $\alpha^{\downarrow i}$ such that $\left(\alpha^{\downarrow i}\right)^{\downarrow j}$ avoids $P$. We rewrite $\alpha^{\downarrow i}$ as

$$
\alpha^{\downarrow i}=\alpha(1) \alpha(2) \ldots \alpha(n-i+1)(n+1) \alpha(n-i+2) \ldots \alpha(n) .
$$

Since $\alpha^{\downarrow k}$ avoids 321, the length $(k-1)$ suffix $\alpha(n-k+2) \ldots \alpha(n)$ of $\alpha$ is increasing, otherwise the suffix $(n+1) \alpha(n-k+2) \ldots \alpha(n)$ of $\alpha^{\downarrow k}$ contains the forbidden pattern 321 . Moreover, since $\alpha^{\downarrow k}$ avoids $(m+1) 12 \ldots m$, we have $k \leq m$, otherwise the same suffix $(n+1) \alpha(n-k+2) \ldots \alpha(n)$ of $\alpha^{\downarrow k}$ contains the forbidden pattern $(m+1) 12 \ldots m$.

If $i=1$ and $k<m$, then the permutation $\left(\alpha^{\downarrow 1}\right)^{\downarrow}(k+1)$ does not contain the pattern $(m+1) 12 \ldots m$ because $k<m$. In addition, $\left(\alpha^{\downarrow 1}\right)^{\downarrow(k+1)}$ contains neither 321 nor $p(p+$ 1) $12 \ldots(p-1)$, otherwise $\alpha^{\downarrow k}$ contains the same pattern.

Moreover, $\left(\alpha^{\downarrow 1}\right)^{\downarrow(k+2)}$ contains at least one of the patterns in $P$, otherwise $\alpha^{\downarrow(k+1)} \in$ $S_{n+1}(P)$. It follows that, in this case, $\chi_{P}(i, k)=k+1$.

If $i=1$ and $k=m$, reasoning in the same manner, the insertion of $(n+2)$ into $\alpha^{\downarrow 1}$ in any site less than or equal to $m$ does not produce patterns in $P$, but the insertion of $(n+2)$ into the $(m+1)$ st site produces the pattern $(m+1) 12 \ldots m$ as a suffix of $\left(\alpha^{\downarrow 1}\right)^{\downarrow(m+1)}$. It follows that, in this case, $\chi_{P}(i, k)=m$.

If $1<i<p$, the insertion of $(n+2)$ into the $(i+1)$ st site of $\alpha^{\downarrow i}$ produces the pattern 321 , whereas the insertion of $(n+2)$ into any site less than or equal to $i$ of $\alpha^{\downarrow i}$ does not produce patterns in $P$, thus in this case, $\chi_{P}(i, k)=i$.

If $i \geq p$, from $i \leq k$ it follows that $p \leq k$, and so the insertion of $(n+2)$ into the $p$ th site of $\alpha^{\downarrow i}$ produces the pattern $p(p+1) 12 \ldots(p-1)$, but the insertion of $(n+2)$ into any site of $\alpha^{\downarrow i}$ less than $p$ does not produce patterns in $P$. Thus in this case $\chi_{P}(i, k)=p-1$.

Notice that, as mentioned in Example 26, particular instances of $m$ and $p$ give classical set of patterns: when $m=p=3, P$ becomes \{321,3421,4123\} investigated in [2, 9] and the corresponding $P$-avoiding permutations are counted by Pell numbers (A000129 in OEIS [13]); and when $p=2$ the obtained $P$-avoiding permutations are counted by the generalized Fibonacci numbers.

We give succession functions for some sets of regular forbidden patterns in Table 2 in Appendix. 


\section{Colored regular patterns}

Not surprisingly, any right-justified set of forbidden patterns $P$ is not necessarily regular: it can happen that $\alpha$ and $\beta$ are $P$-avoiding permutations having the same number of active sites, but the insertion of the next largest value into the $i$ th active site of both $\alpha$ and $\beta$ yields permutations with different numbers of active sites. This section is devoted to the investigation of a particular class of such right-justified forbidden patterns that we call, following Barcucci et al. [2], colored regular forbidden patterns. For such forbidden patterns we develop corresponding succession functions and explicit them for two sets of forbidden patterns: that in Example 251 and the second one in Example 22.

Let $\alpha$ be a permutation with $k$ active sites belonging to $S(P)$, with $P$ a right-justified set of forbidden patterns, and let $i$ be an active site of $\alpha, 1 \leq i \leq k$. Suppose that it exists a procedure coloring by integer values the permutations in $S(P)$, so that: (i) the number of active sites of $\alpha^{\downarrow i}$ does not depend on $\alpha$ but only on the three parameters $i, k$ and the color $c$ of $\alpha$; and (ii) the color of $\alpha^{\downarrow i}$ in turn, depends only on $i, k$ and $c$. In this case we extend the function $\chi_{P}$ in the previous section so that it transforms the triple $(i, k, c)$ into a pair of integers: the number of active sites and the color of $\alpha^{\downarrow i}$. In order to anchor the recursivity we set the color of the length zero permutation $\epsilon$ to 0 , and we have the next definition.

Definition 3 (c-regular pattern). A set $P$ of right-justified forbidden patterns is called colored regular (c-regular for short) if for any $\alpha \in S(P)$,

- $\alpha$ has its first site active, and

- if $\alpha$ has $k$ active sites and color $c$, then for any $i, 1 \leq i \leq k$

- the number of active sites of $\alpha^{\downarrow i}$ depends only on $i, k$ and $c$, and we denote this number by $\mu_{P}(i, k, c)$,

- the color of $\alpha^{\downarrow i}$ depends as above only on $i, k$ and $c$, and we denote this color by $\nu_{P}(i, k, c)$.

In this case, the succession function $\chi_{P}=\left(\mu_{P}, \nu_{P}\right)$ becomes:

$$
\chi_{P}=\left(\mu_{P}, \nu_{P}\right):\left\{(i, k, c) \mid k \in \mathbb{N}^{+}, 1 \leq i \leq k, c \in C\right\} \rightarrow \mathbb{N}^{+} \times C,
$$

where $C \subset \mathbb{N}$ is the set of colors.

Notice that regular patterns are particular c-regular patterns, where the set of colors collapses to $\{0\}$.

Now we consider the set of right-justified forbidden patterns $P$ in Example 2 15, and the next theorem shows that $P$ is a c-regular set of forbidden patterns by giving explicitly its colored succession function. We postpone its proof after giving some technical results.

Theorem 3. The colored succession function for the set of forbidden patterns $P=$ $\{312,2431,(p+1) p \ldots 21\}, p \geq 2$, is $\chi_{P}(i, k, c)=\left(\mu_{P}(i, k, c), \nu_{P}(i, k, c)\right)$, with set of colors $\{0,1\}$ and:

$$
\mu_{P}(i, k, c)= \begin{cases}i+1 & \text { if } i=1 \text { or }(i=k \text { and } c=0 \text { and } k<p) \\ i & \text { otherwise, }\end{cases}
$$


and

$$
\nu_{P}(i, k, c)= \begin{cases}0 & \text { if } i=1 \text { or } \\ 1 & \text { otherwise. }\end{cases}
$$

\section{Lemma 1.}

1. If $\alpha=\alpha(1) \alpha(2) \ldots \alpha(n)$ is a length $n$ permutation avoiding 312, and $i$ is such that $\alpha(i)=n$, then the suffix $\alpha(i) \alpha(i+1) \ldots \alpha(n)$ of $\alpha$ is decreasing.

2. Let $P$ be the set of forbidden patterns in Theorem 3. If $\alpha \in S_{n}(P)$ has $k$ active sites, and $\beta=\alpha^{\downarrow i} \in S_{n+1}(P)$ for some $i, 1 \leq i \leq k$, then $\beta$ has either $i$ or $i+1$ active sites.

Proof. For the first point, since $\alpha$ avoids 312 and $\alpha(i)=n$ is the largest value of the suffix $\alpha(i) \alpha(i+1) \ldots \alpha(n)$, it follows that this suffix is decreasing.

For the second point, the insertion of $(n+2)$ into the $i$ th site of $\beta=\alpha^{\downarrow i}$ produces no patterns in $P$ (the entries $(n+1)$ and $(n+2)$ are consecutive in $\beta$ ), and the insertion of $(n+2)$ into the $(i+2)$ nd site of $\beta$ produces the forbidden pattern 312 , and since $P$ is a right-justified set of forbidden patterns the result follows.

The following result is a direct consequence of the second point of the previous lemma, and we state it in the next corollary in order to refer to it later.

Corollary 2. Let $P$ be the set of forbidden patterns in Theorem 3. If $\alpha \in S_{n}(P), n \geq 1$, has $k$ active sites, then $\alpha=\lambda^{\downarrow k}$ or $\alpha=\lambda^{\downarrow(k-1)}$ for some $\lambda \in S_{n-1}(P)$.

As one can see below, the pattern 231 is of particular interest for the definition of the color of a permutation in Theorem 3. If in an arbitrary permutation $\alpha \in S_{n}, n$ is involved in an occurrence of the pattern 231, then $n$ plays the role of 3 in this occurrence and we have the following easy to understand result.

To each permutation $\alpha$ in $S_{n}(P), P=\{312,2431,(p+1) p \ldots 21\}$, we associate an integer $d(\alpha) \in\{0,1\}$ as: $d(\alpha)$ is 0 if and only if at least one of the following two conditions is fulfilled: $n$ is not involved in an occurrence of the pattern 231 in $\alpha$, or the length $p$ suffix of $\alpha$ is decreasing. Equivalently, $d(\alpha)$ is 1 if and only if $n$ plays the role of 3 in an occurrence of the pattern 231 in $\alpha$ and the length $p$ suffix of $\alpha$ is not decreasing. Thus $d$ is a function $d: S(P) \rightarrow\{0,1\}$.

Proof of Theorem 3. Actually, we will prove by induction on $n$ the following:

(1) the color of a permutation $\alpha \in S(P)$ defined in Theorem 3 by means of $\nu_{P}$ is $d(\alpha)$;

(2) the statement of Theorem 3 .

If $n=0$, then (1) and (2) trivially hold.

Proof of (1): Supposing that (1) and (2) are satisfied by length $n$ permutations, $n \geq 0$, we prove (1) for length $n+1$ permutations.

Let $\alpha$ be a permutation in $S_{n}(P)$ with $k$ active sites, $n \geq 0$.

First we show that $i=1$, or $i=k$ and $d(\alpha)=0$ implies $d\left(\alpha^{\downarrow i}\right)=0$.

If $i=1$, clearly $(n+1)$ is not involved in an occurrence of 231 in $\alpha^{\downarrow 1}$, so $d\left(\alpha^{\downarrow 1}\right)=0$.

If $i=k>1$ and $d(\alpha)=0$, we have $n>0$, and we distinguish two cases. 
- $k=p$. By Lemma 1,1, the length $p$ suffix of $\alpha^{\downarrow i}=\alpha^{\downarrow p}$ is decreasing and so $d\left(\alpha^{\downarrow i}\right)=0$.

- $k<p$. By Corollary 2 it follows that $\alpha=\lambda^{\downarrow(k-1)}$ or $\alpha=\lambda^{\downarrow k}$ for some $\lambda \in S_{n-1}(P)$. If $\alpha=\lambda^{\downarrow(k-1)}$, since $\alpha$ has $k$ active sites and $(n+1)$ and $n$ are conscutive entries in $\alpha^{\downarrow k}=\left(\lambda^{\downarrow(k-1)}\right)^{\downarrow k}$ it follows that $n$ is not involved in an occurrence of 231 in $\alpha$ (otherwise $\alpha^{\downarrow k}$ contains the forbidden pattern 2431), and $(n+1)$ is not involved in an occurrence of 231 in $\alpha^{\downarrow k}$, so $d\left(\alpha^{\downarrow k}\right)=0$. But $\alpha=\lambda^{\downarrow k}$ is not possible, indeed $\alpha=\lambda^{\downarrow k}$ implies either:

$-\lambda$ has $j$ active sites with $j>k$, and in this case $n$ is involved in an occurrence of 231 in $\alpha$ which is in contradiction with $d(\alpha)=0$; or

$-\lambda$ has $($ as $\alpha) k$ active sites which implies that the number of active sites of $\alpha$ follows the second rule in the definition of $\mu_{P}$ in the statement of the present theorem, which in our case happens when the the color of $\lambda$ is 1 , hence the color of $\alpha=\lambda^{\downarrow k}$ follows the second rule in the definition of $\nu_{P}$, which again leads to a contradiction.

Conversely, it is routine to check that when $i>1$ and $(i<k$ or $d(\alpha)=1)$ the insertion of $(n+1)$ into the $i$ th active site of $\alpha$ produces a new occurrence of the pattern 231, but not a length $p$ decreasing suffix, and so $d\left(\alpha^{\downarrow}\right)=1$.

Proof of (2): Supposing that (1) and (2) are satisfied by length $n$ permutations, $n \geq 0$, we prove (2) for length $n+1$ permutations. In light of (1) it is enough to show that the number of active sites of a permutation in $S_{n+1}(P)$ is that specified by $\mu_{p}$ and considering its color given by the function $d$.

Let $\alpha$ be a permutation in $S_{n}(P)$ with $k$ active sites and color $c$, and let $\beta=\alpha^{\downarrow}$ be the permutation obtained from $\alpha$ by inserting $(n+1)$ into its $i$ th active site, $1 \leq i \leq k$. By Lemma 12, $\beta$ has $i$ or $i+1$ active sites. If the condition ' $i=1$ or $(i=k$ and $c=0$ and $k<p)^{\prime}$ is satisfied, then the insertion of $(n+2)$ into the $(i+1)$ st site of $\beta$ produces no forbidden pattern, and so $\beta$ has $i+1$ active sites.

By contrast, if the above condition is violated, then the insertion of $(n+2)$ into the $(i+1)$ st site of $\beta$ produces a forbidden pattern. To prove this, it is enough to show that $\beta^{\downarrow}(i+1)$ contains a forbidden pattern if $i$ satisfies one of the following conditions: (i) $i>1$ and $(i<k$ or $c=1)$, or (ii) $i=k=p$.

If $i>1$ and $(i<k$ or $c=1)$, then $(n+1)$ plays the role of 3 in an occurrence of the pattern 231 in $\alpha^{\downarrow i}$ (see the last part of the proof of (1)), and thus $\beta^{\downarrow(i+1)}$ contains the forbidden pattern 2431 .

If $i=k=p$, then $\beta^{\downarrow(i+1)}$ contains the forbidden pattern $(p+1) p \ldots 1$.

In particular, when $p=3$ the set of forbidden patterns in Theorem 3 becomes $P=$ $\{312,2431,4321\}$ and the corresponding counting sequence is the binomial transform of Padovan sequence (A034943 in OEIS [13]). An illustration of the underlying tree of the succession function for this set of forbidden patterns is given in Figure 1. It turns out that in this particular case the number of active sites of a permutation in $S_{n}(P)$ is either 2 or 3 (except for the empty permutation $\epsilon$ ), and we have the next corollary.

Corollary 3. The succession function for the set of forbidden patterns $P=\{312,2431,4321\}$ is given by: 


$$
\mu_{P}(i, k, c)= \begin{cases}2 & \text { if } i=1 \text { or } i=2 \text { and }(c=1 \text { or } k=3) \\ 3 & \text { otherwise, }\end{cases}
$$

and

$$
\nu_{P}(i, k, c)= \begin{cases}0 & \text { if } i=1 \text { or }(i=k \text { and } c=0) \\ 1 & \text { otherwise. }\end{cases}
$$

Notice that the binomial transform of Padovan sequence also counts permutations avoiding $\{321,2413,3142\}$, see [14]. However, by Theorem[1, this set of forbidden patterns is not right-justified whereas $P=\{312,2431,4321\}$ is regular.

In the same vein, we give below without proof the colored succession function for the second set of patterns in Example 22, Its proof is similar with that of Theorem 3 when the variable length pattern is omitted.

Proposition 1. The succession function for the set of forbidden patterns $P=\{312,2431\}$ is $\chi_{P}(i, k, c)=\left(\mu_{P}(i, k, c), \nu_{P}(i, k, c)\right)$, with

$$
\mu_{P}(i, k, c)= \begin{cases}i+1 & \text { if } i=1 \text { or }(i=k \text { and } c=0) \\ i & \text { otherwise, }\end{cases}
$$

and

$$
\nu_{P}(i, k, c)= \begin{cases}0 & \text { if } i=1 \text { or } \\ 1 & \text { otherwise. }\end{cases}
$$

We give succession functions for some sets of c-regular forbidden patterns in Table 3 in Appendix.

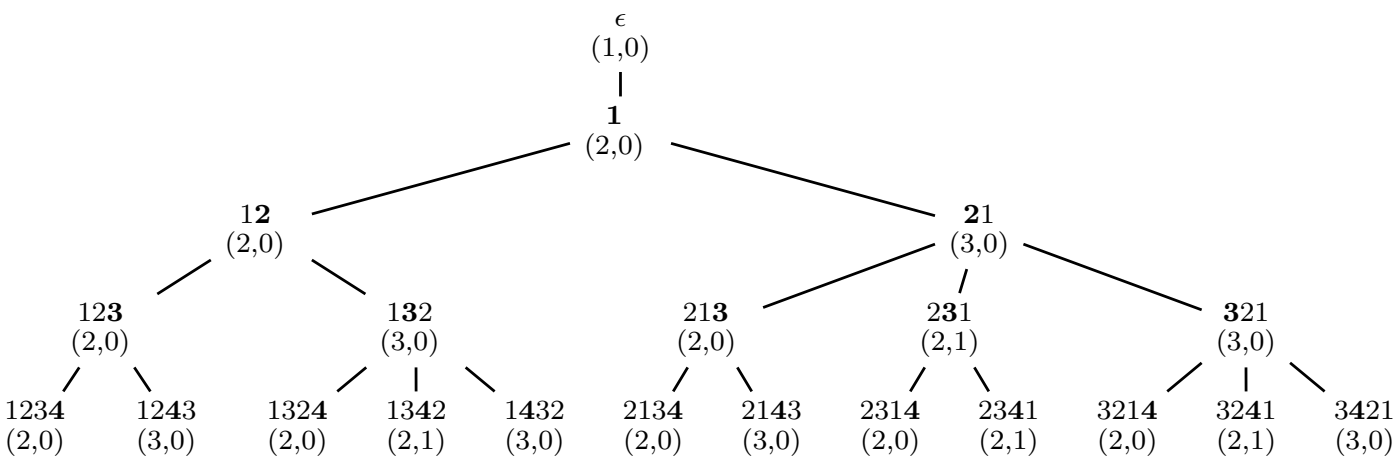

Figure 1: The first levels of the tree induced by the succession function in Theorem 3 when $p=3$ (Corollary 3). Each node of the tree is labeled $\underset{(k, c)}{\alpha}$, with $\alpha$ a permutation in $S_{n}(P), 0 \leq n \leq 4$, $P=\{312,2431,4321\}, k$ the number of active sites and $c$ the color of $\alpha$.

\section{$5 \quad$ Efficient generating algorithms}

In this section, we present exhaustive generating algorithms for permutations avoiding a set of forbidden regular and c-regular patterns and we show that they are efficient. 
For a set of forbidden patterns $P$ and for $\alpha \in S_{n}(P)$, since $\alpha^{\downarrow(i+1)}=\left(\alpha^{\downarrow i}\right)^{\leftarrow}$, we have that $\alpha^{\downarrow i}$ and $\alpha^{\downarrow(i+1)}$ differ by a transposition of two adjacent entries, one of them being $(n+1)$. In our algorithms we represent permutations $\alpha \in S_{n}$ by length $n$ global arrays. The insertion of an element into an array is not an efficient operation, except when a next largest entry is inserted after the last entry of the array. By contrast, the transposition of two adjacent elements requires only a constant time and we express insertions by iterating transpositions of adjacent entries, and this is crucial for the efficiency of our generating algorithms. In the algorithms in Table 1, $\alpha \cdot(i, j)$ is the permutation obtained from $\alpha$ by transposing the entries in positions $i$ and $j$; and $[\alpha$, length $]$ is the permutation in $S_{\text {length }}(P)$ obtained from $\alpha \in S_{\text {length-1 }}(P)$ by inserting the integer length after the last entry of $\alpha$, that is the permutation $\alpha^{\downarrow 1}$.

Given an explicit implementation of the succession function $\chi_{P}$, the generating algorithms in Table 1 produce exhaustively the length $n P$-avoiding permutations, for a set of regular or c-regular forbidden patterns $P$. In particular, the algorithm (a) (which is first mentioned by Duckes et al. [8] in the context of Gray code generation) mimes the succession rules given after Definition 2 , and tree induced by the recursive calls of the algorithm (b) for the permutations in Corollary 3 with $n \leq 4$ is depicted in Figure 1.

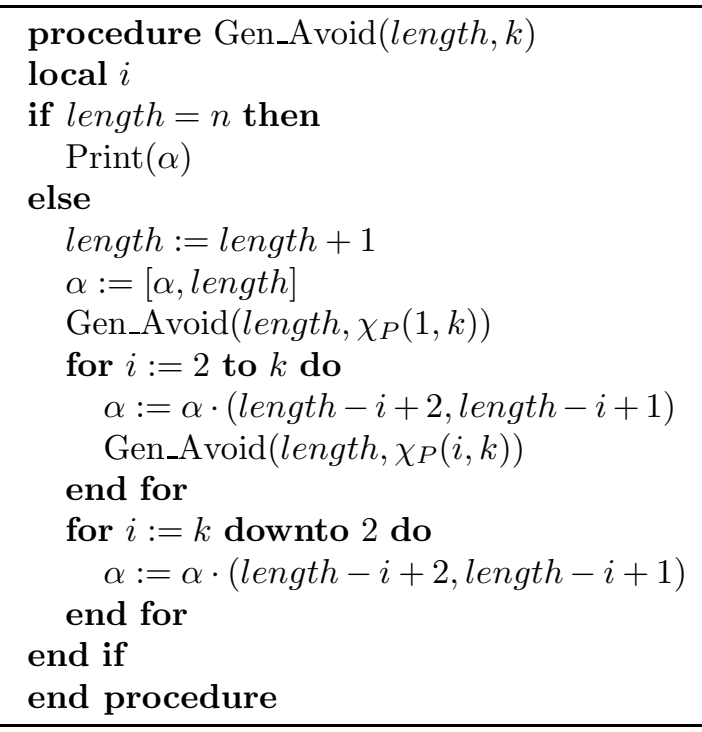

(a)

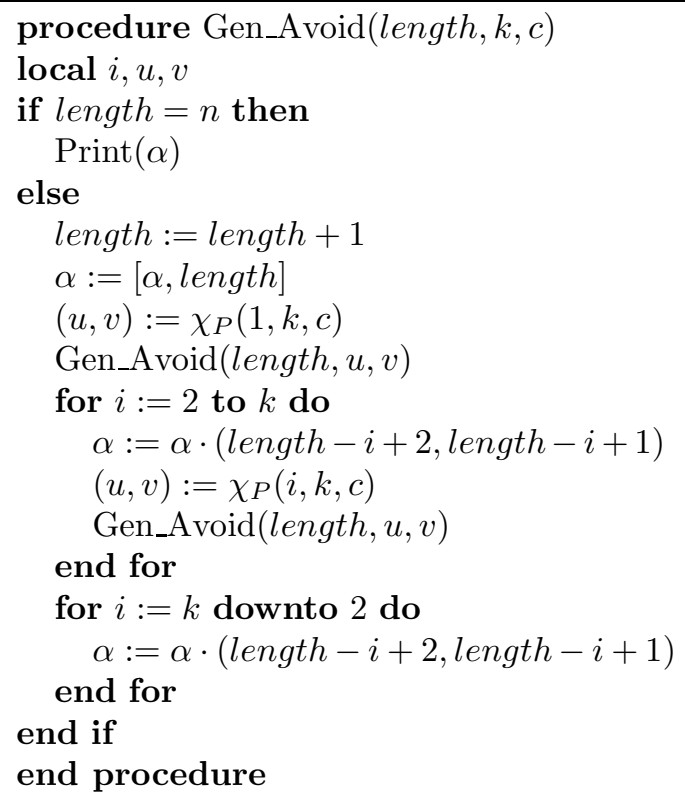

(b)

Table 1: (a) Algorithm for generating permutations avoiding: (a) a regular pattern characterized by the succession function $\chi_{P}$, with the initial call Gen_Avoid $(0,1)$; and (b) a c-regular pattern characterized by the succession function $\chi_{P}$, with the initial call Gen_Avoid $(0,1,0)$. In both cases the initial permutation is the length zero permutation $\epsilon$.

A recursive generating algorithm is said to run in constant amortized time (CAT) if it generates each object in $O(1)$ time, in amortized sense. Such an algorithm is also called a CAT algorithm. The following CATness principle is due to Frank Ruskey.

Proposition 2. ([12]) A recursive generating algorithm is a CAT one if it satisfies the 
following properties:

- Each recursive call generates at least one object (there is no dead-end recursive call);

- The amount of computation in each recursive call is proportional to the degree of the call (that is, the number of subsequent recursive calls produced by the current call);

- The number of recursive calls having degree one (if any) is $O(N)$, where $N$ is the number of generated objects.

Let $P$ be a set of regular or c-regular forbidden patterns, and $\alpha \in S_{n}(P), n \geq 0$. By the defintion of regularity, $\alpha^{\downarrow 1}$ belongs to $S_{n+1}(P)$, and if $\alpha^{\downarrow 1}$ has only one active site (or equivalenty, $\left(\alpha^{\downarrow 1}\right)^{\downarrow 2}$ contains a pattern in $P$ ), then there is a length $k \geq 2$ permutation $\tau=\tau(1) \tau(2) \ldots \tau(k-2) k(k-1)$ belonging to $P$.

The number of recursive calls produced by a current call of our algorithms is given by $\chi_{P}$, and combining Proposition 2 with the considerations above we have the following theorem.

Theorem 4. If $P$ is a set of regular or c-regular forbidden patterns for which the corresponding succession function $\chi_{P}$ can be computed in constant time, and $P$ does not contain patterns $\tau$ of the form $\tau(1) \tau(2) \ldots \tau(k-2) k(k-1), k \geq 2$, then the algorithms in Table 1 generate in constant amortized time the set $S_{n}(P), n \geq 0$.

In Tables 2 and 3 in Appendix we list several regular and c-regular sets of forbidden patterns satisfying Theorem 4. For some of them, the corresponding succession functions are given in Sections 3 and 4 of the present paper.

The generating order of our algorithms is not the lexicographical one, and we have the next proposition.

Proposition 3. Let $L_{n}(P), n \geq 0$, be the ordered list for the set $S_{n}(P)$ produced by algorithms in Table 1. Then $\alpha$ precedes $\beta$ in this list if either $n \geq 1$ and $\alpha^{\prime}$ precedes $\beta^{\prime}$ in $L_{n-1}(P)$, where $\alpha^{\prime}$ and $\beta^{\prime}$ are the permutations obtained from $\alpha$ and $\beta$ by erasing their largest element $n$; or $i<j$, where $i$ and $j$ are the positions (from right to left) of $n$ in $\alpha$ and $\beta$.

Finally, if for a set of right-justified forbidden patterns $P, P^{r}$ (resp. $P^{c}$ ) denotes the set of patterns obtained by reversing (resp. complementing) each pattern in $P$ (see for example [10] for the definition of these two operations), then our algorithms can easily be adapted to generate $S_{n}\left(P^{r}\right)$ and $S_{n}\left(P^{c}\right)$ provided they generate $S_{n}(P)$.

\section{Acknowledgment}

This research is funded by Vietnam National Foundation for Science and Technology Development (NAFOSTED) under grant number 102.01-2016.05. A part of this manuscript was accomplished when the second author was visiting the Vietnam Institute for Advanced Study in Mathematics (VIASM). 


\section{References}

[1] S. Bacchelli, E. Barcucci, E. Grazzini, and E. Pergola. Exhaustive generation of combinatorial objects by ECO. Acta Informatica, 40:585 - 602, 2004.

[2] E. Barcucci, A. Bernini, and M. Poneti. From Fibonacci to Catalan permutations. Pu.M.A., Volume 17, Issue No. 1-2, 2006.

[3] E. Barcucci, A. Del Lungo, E. Pergola, and R. Pinzani. ECO: a methodology for the enumeration of combinatorial objects. Journal of Difference Equations and Applications, 5:435-490, 1999.

[4] J.-L. Baril and P.T. Do. ECO-generation for $p$-generalized Fibonacci and Lucas permutations. Pu.M.A., 17:1 - 19, 2006.

[5] T. Chow and J. West. Forbidden sequences and Chebyshev polynomials. Discrete Mathematics, 204:119 - 128, 1999.

[6] P.T. Do. Arbres de génération et génération exhausitive. PHD Thesis, University of Burgundy, France, 2008.

[7] P.T. Do, T.T.H. Tran, and V. Vajnovszki. Right-justified characterization and succession functions for exhaustive generation of colored regular pattern avoiding permutations. In In: BlÃ mer J., Kotsireas I., Kutsia T., Simos D. (eds) Mathematical Aspects of Computer and Information Sciences. MACIS 2017. Lecture Notes in Computer Science, 10693:306-319, 2017.

[8] W.M.B. Dukes, M.F. Flanagan, T. Mansour, and V. Vajnovszki. Combinatorial Gray codes for classes of pattern avoiding permutations. Theoretical Computer Science, 396:35-49, 2008.

[9] O. Guibert. Combinatoire des permutations à motifs exclus en liaison avec mots, cartes planaires et tableaux de Young. PHD Thesis, Université Bordeaux 1, 1995.

[10] S. Kitaev. Patterns in Permutations and Words. Springer, 2011.

[11] D.E. Knuth. The Art of Computer Programming, Volume 4A: Combinatorial Algorithms, Part 1. Addison-Wesley, 2011.

[12] F. Ruskey. Combinatorial Generation. In preparation, version 2008.

[13] N.J.A. Sloane. The on-line encyclopedia of integer sequences (OEIS). Published electronically at http://oeis.org.

[14] V. Vatter. Finding regular insertion encodings for permutation classes. Journal of Symbolic Computation, 47(3):259-265, 2012.

[15] J. West. Generating trees and the Catalan and Schröder numbers. Discrete Mathematics, 146:247 - 262, 1994. 


\section{Appendix}

\begin{tabular}{|c|c|c|}
\hline Counting sequence/class & $P$ & $\chi_{P}(i, k)$ \\
\hline $2^{n-1}[2]$ & $\{321,312\}$ & 2 \\
\hline \multirow{2}{*}{$\begin{array}{l}\text { Pell numbers [2, 9] } \\
\text { (A000129 in OEIS [13]) }\end{array}$} & $\begin{array}{l}\{321,3412,4123\} \\
\text { particular instance of Theorem } 2\end{array}$ & $\begin{array}{ll}3 & \text { if } i=1 \\
2 & \text { otherwise. }\end{array}$ \\
\hline & $\{312,4321,3421\}$ & $\begin{array}{ll}3 & \text { if } i=2 \\
2 & \text { otherwise. }\end{array}$ \\
\hline \multirow{3}{*}{$\begin{array}{l}\text { Bisection of Fibonacci sequence [2, 9] } \\
(\text { A001519 in OEIS [13]) }\end{array}$} & $\begin{array}{l}\{321,3412\} \\
\text { particular instance of Theorem } 2\end{array}$ & $\begin{array}{ll}k+1 & \text { if } i=1 \\
2 & \text { otherwise. }\end{array}$ \\
\hline & $\begin{array}{l}\{321,4123\} \\
\text { particular instance of Theorem } 2\end{array}$ & $\begin{array}{ll}3 & \text { if } i=1 \\
i & \text { otherwise. }\end{array}$ \\
\hline & $\{312,4321\}$ & $\begin{array}{ll}3 & \text { if } k=3 \text { and } i=3 \\
i+1 & \text { otherwise. }\end{array}$ \\
\hline \multirow{2}{*}{$\begin{array}{l}\text { Catalan numbers [15] } \\
(\text { A000108 in OEIS [13]) }\end{array}$} & $\{312\}$ & $i+1$ \\
\hline & $\{321\}$ & $\begin{array}{ll}k+1 & \text { if } i=1 \\
i & \text { otherwise. }\end{array}$ \\
\hline $\begin{array}{l}\text { Schröder numbers }[9] \\
(\text { A006318 in OEIS }[13)\end{array}$ & $\{4123,4213\}$ & $\begin{array}{ll}k+1 & \text { if } i=k-1 \text { or } i=k \\
i+2 & \text { otherwise. }\end{array}$ \\
\hline $\begin{array}{l}\text { Fibonacci numbers }[4 \\
(\text { A000045 in OEIS [13]) }\end{array}$ & $\begin{array}{l}\{321,231,312\} \\
\text { particular instance of Theorem } 2\end{array}$ & $\begin{array}{ll}1 & \text { if } i=2 \\
2 & \text { otherwise. }\end{array}$ \\
\hline \multirow{3}{*}{$\begin{array}{l}\text { A pattern of length } 3 \text { and } \\
\text { a variable length pattern }\end{array}$} & $\begin{array}{l}\{321,(p+1) 12 \ldots p\}[2,5] \\
\quad \text { particular instance of Theorem } 2\end{array}$ & $\begin{array}{ll}k+1 & \text { if } i=1 \text { and } k<p \\
p & \text { if } i=1 \text { and } k=p \\
i & \text { otherwise. }\end{array}$ \\
\hline & $\begin{array}{l}\{321, p(p+1) 12 \ldots(p-1)\} 2,5 \\
\quad \text { particular instance of Theorem } 2\end{array}$ & $\begin{array}{ll}k+1 & \text { if } i=1 \\
i & \text { if } 1<i<p-1 \\
p-1 & \text { otherwise. }\end{array}$ \\
\hline & $\{312,(p+1) p \ldots 21\}$ & $\begin{array}{ll}p & \text { if } k=p \text { and } i=p \\
i+1 & \text { otherwise. }\end{array}$ \\
\hline $\begin{array}{l}\text { A pattern of length } 3, \\
\text { a pattern of length } 4 \text { and } \\
\text { a variable length pattern }\end{array}$ & $\begin{array}{l}\{321,3412,(p+1) 12 \ldots p\}[2] \\
\quad \text { particular instance of Theorem } 2\end{array}$ & $\begin{array}{ll}k+1 & \text { if } i=1 \text { and } k<p \\
p & \text { if } i=1 \text { and } k=p \\
2 & \text { otherwise. }\end{array}$ \\
\hline $\begin{array}{l}\text { Generalized Fibonacci } \\
\text { numbers }\end{array}$ & $\begin{array}{l}\{321,231,(p+1) 12 \ldots p\}[2] \\
\quad \text { particular instance of Theorem } 2\end{array}$ & $\begin{array}{ll}k+1 & \text { if } i=1 \text { and } k<p \\
k & \text { if } i=1 \text { and } k=p \\
1 & \text { otherwise. }\end{array}$ \\
\hline \multirow[t]{2}{*}{$\begin{array}{l}\text { A pattern of length } 3 \text { and } \\
\text { two variable length patterns }\end{array}$} & $\begin{array}{l}\{321, p(p+1) 12 \ldots(p-1),(p+1) 12 \ldots p\}[2] \\
\quad \text { particular instance of Theorem } 2\end{array}$ & $\begin{array}{ll}k+1 & \text { if } i=1 \text { and } k<p \\
p & \text { if } i=1 \text { and } k=p \\
p-1 & \text { if } i=p \text { and } k=p \\
i & \text { otherwise. }\end{array}$ \\
\hline & $\begin{array}{l}\{321, p(p+1) 12 \ldots(p-1),(m+1) 12 \ldots m\} \\
\quad \text { Theorem } 2\end{array}$ & $\begin{array}{ll}k+1 & \text { if } i=1 \text { and } k<m \\
m & \text { if } i=1 \text { and } k=m \\
i & \text { if } 1<i<p \\
p-1 & \text { otherwise. }\end{array}$ \\
\hline
\end{tabular}

Table 2: A sample of regular forbidden patterns $P$. Their succession function (last column) is either folklore or easy to check, or given in the corresponding reference. 


\begin{tabular}{|c|c|c|c|}
\hline Counting sequence/class & $P$ & $\mu_{P}(i, k, c)$ & $\nu_{P}(i, k, c)$ \\
\hline $\begin{array}{l}\text { Bisection of Fibonacci sequence } \\
(\text { A001519] in OEIS [13]) }\end{array}$ & $\begin{array}{l}\{312,2431\} \\
\text { Proposition } 1\end{array}$ & $\begin{array}{ll}i+1 & \text { if } i=1 \text { or } \\
& (i=k \text { and } c=0) \\
i & \text { otherwise. }\end{array}$ & $\begin{array}{ll}0 & \text { if } i=1 \text { or } \\
& (i=k \text { and } c=0) \\
1 & \text { otherwise. }\end{array}$ \\
\hline $\begin{array}{l}\text { Binomial transform of } \\
\text { Padovan sequence } \\
\text { (A034943 in OEIS [13]) }\end{array}$ & $\begin{array}{l}\{312,2431,4321\} \\
\text { Corollary } 3\end{array}$ & $\begin{array}{c}2 \quad \text { if } i=1 \text { or } i=2 \text { and } \\
\quad(c=1 \text { or } k=3) \\
3 \quad \text { otherwise. }\end{array}$ & $\begin{array}{cc}0 & \text { if } i=1 \text { or } \\
& (i=k \text { and } \\
& c=0) \\
1 & \text { otherwise. }\end{array}$ \\
\hline $\begin{array}{l}\text { Generalized Fibonacci } \\
\text { numbers }\end{array}$ & $\{321,312,23 \ldots(p+1) 1\}$ & $\begin{array}{cc}1 & \text { if } c=p-2 \\
& \text { and } i=2 \\
2 & \text { otherwise. }\end{array}$ & $\begin{array}{lr}0 & \text { if } i=1 \text { or } \\
& c=p-2 \\
c-1 & \text { otherwise. }\end{array}$ \\
\hline \multirow[t]{2}{*}{$\begin{array}{l}\text { A 3-length pattern, } \\
\text { a 4-length pattern } \\
\text { and a variable } \\
\text { length pattern }\end{array}$} & $\{321,4123,34 \ldots(p+1) 12\} 2$ & $\begin{array}{l}2 \quad \text { if } i=2 \text { or }(i=3 \\
\quad \text { and } c=p-3) \\
3 \text { otherwise. }\end{array}$ & $\begin{array}{ll}c+1 & \text { if } i=3 \\
& \text { and } c<p-3 \\
0 & \text { otherwise. }\end{array}$ \\
\hline & $\begin{array}{l}\{312,2431,(p+1) p \ldots 21\} \\
\quad \text { Theorem } 3\end{array}$ & $\begin{array}{ll}i+1 \quad \text { if } i=1 \text { or } \\
& (i=k<p \text { and } \\
& c=0) \\
i & \text { otherwise. }\end{array}$ & $\begin{array}{ll}0 & \text { if } i=1 \text { or } \\
& (i=k<p \text { and } \\
& c=0) \\
1 & \text { otherwise. }\end{array}$ \\
\hline
\end{tabular}

Table 3: A sample of c-regular forbidden patterns $P$ together with the $\chi_{P}(i, k, c)=$ $\left(\mu_{P}(i, k, c), \nu_{P}(i, k, c)\right)$ functions. 